\title{
Preprocessing of solar vector magnetograms for force-free magnetic field extrapolation
}

\author{
M. Fuhrmann ${ }^{1}$, N. Seehafer ${ }^{1}$, and G. Valori ${ }^{2}$ \\ 1 Institut für Physik, Universität Potsdam, Am Neuen Palais 10, 14469 Potsdam, Germany \\ e-mail: fuhrmann@agnld.uni-potsdam.de; seehafer@uni-potsdam.de \\ 2 Astrophysikalisches Institut Potsdam, An der Sternwarte 16, 14482 Potsdam, Germany \\ e-mail: gvalori@aip.de
}

Received 9 August 2007 / Accepted 7 September 2007

\begin{abstract}
Context. Reliable measurements of the solar magnetic field are restricted to the phoptosphere. As an alternative to measurements, the field in the higher layers of the atmosphere is calculated from the measured photospheric field, mostly under the assumption that it is force-free. However, the magnetic field in the photosphere is not force-free. Moreover, most methods for the extrapolation of the photospheric magnetic field into the higher layers prescribe the magnetic vector on the whole boundary of the considered volume, which overdetermines the force-free field. Finally, the extrapolation methods are very sensitive to small-scale noise in the magnetograph data, which, however, if sufficienly resolved numerically, should affect the solution only in a thin boundary layer close to the photosphere.

Aims. A new method for the preprocessing of solar photospheric vector magnetograms has been developed that, by improving their compatibility with the condition of force-freeness and removing small-scale noise, makes them more suitable for extrapolations into three-dimensional nonlinear force-free magnetic fields in the chromosphere and corona.

Methods. A functional of the photospheric field values is minimized whereby the total magnetic force and the total magnetic torque on the considered volume above the photosphere, as well as a quantity measuring the degree of small-scale noise in the photospheric boundary data, are simultaneously made small. For the minimization, the method of simulated annealing is used and the smoothing of noisy magnetograph data is attained by windowed median averaging.

Results. The method was applied to a magnetogram derived from a known nonlinear force-free test field to which an artificial noise had been added. The algorithm recovered all main structures of the magnetogram and removed small-scale noise. The main test was to extrapolate from the noisy photospheric vector magnetogram before and after the preprocessing. The preprocessing was found to significantly improve the agreement of the extrapolated with the exact field.
\end{abstract}

Key words. Sun: magnetic fields - Sun: atmosphere - magnetohydrodynamics (MHD)

\section{Introduction}

Reliable measurements of the solar magnetic field are still restricted to the level of the photosphere. The situation here is improving only very slowly due to elementary difficulties in unambiguously deriving the magnetic field from polarimetric measurements in chromospheric or coronal spectral lines. As an alternative to measurements in these superphotospheric layers, for about half a century attempts have been made to calculate the field there from the measured photospheric field using physically plausible assumptions. The procedure is known as magnetic field extrapolation.

The simplest assumption was to consider the magnetic field $\boldsymbol{B}$ above the photosphere as a vacuum field or current-free field or potential field, satisfying $\nabla \times \boldsymbol{B}=\mathbf{0}$. Potential field models were devised both for the field above limited photospheric areas, in particular active regions (Schmidt 1964; Teuber et al. 1977), and for the global field above the spherical photosphere (Schatten et al. 1969; Altschuler \& Newkirk 1969).

A next step was to include chromospheric and coronal electric currents into the models. Such currents are needed as the energy source for explosive events like flares and coronal mass ejections. For typical plasma parameters in the superphotospheric parts of active regions, except for the times of the explosive events, the magnetic energy density dominates over the thermal, kinetic and gravitational energy densities. This implies that, if appreciable currents are present, these must be aligned with the magnetic field, otherwise the resulting Lorentz forces could not be balanced by the non-magnetic forces. Thus, the magnetic field must be approximately force-free, characterized by the equations

$$
\begin{aligned}
& \nabla \times \boldsymbol{B}=\alpha(\boldsymbol{r}) \boldsymbol{B}, \\
& \nabla \cdot \boldsymbol{B}=0,
\end{aligned}
$$

where $\alpha(\boldsymbol{r})$ denotes a scalar function of position $\boldsymbol{r}$ which, because of Eq. (2), is constant along the magnetic field lines. This approximation is presumably valid from the upper chromosphere up to heights of $\sim R_{\odot}$ above the level of the photosphere in the corona (see Gary 2001, for a careful study of the height variation of the plasma $\beta$, i.e. the ratio between the thermal and magnetic pressures or energy densities).

Solutions to Eqs. (1)-(2) for the special case of a spatially constant $\alpha$ were given by Nakagawa \& Raadu (1972), 
Chiu \& Hilton (1977), Seehafer (1978), Alissandrakis (1981), and Semel (1988); see also reviews by Seehafer \& Staude (1983), Gary (1989), and Sakurai (1989). The practical application to solar active regions has been largely confined to these constant- $\alpha$ or linear force-free fields, whose determination leads to boundary value problems for a linear partial differential equation. The extrapolations with constant- $\alpha$ force-free fields, as well as those with current-free fields, have provided new insights into the physics of the activity phenomena. In particular, results were obtained on magnetic topologies favourable for flares, and on magnetic and current helicities. One example is the potential role of separatrices made up of magnetic field lines touching the photospheric boundary from above for the formation of electric current sheets, and the fast release of stored magnetic energy by magnetic reconnection (Seehafer 1986). These topological elements have been termed bald patches (Titov et al. 1993; Bungey et al. 1996; Titov \& Démoulin 1999). Another finding is the hemispheric sign rule for the current helicity $\boldsymbol{B} \cdot(\nabla \times \boldsymbol{B})$, namely that this quantity is predominantly negative in the northern hemisphere and positive in the southern hemisphere (Seehafer 1990).

The extrapolation methods for current-free and constant- $\alpha$ force-free fields require only line-of-sight magnetograms (with $\alpha$ remaining as a free parameter in the case of the constant- $\alpha$ fields). Since the early $80 \mathrm{~s}$, different methods for calculating non-constant- $\alpha$ or nonlinear force-free fields from photospheric vector magnetograms have been proposed, and presently great efforts are being made to refine them and to improve their applicability. These methods include:

- Direct vertical integration (Wu et al. 1985, 1990; Cuperman et al. 1990; Démoulin et al. 1992; Song et al. 2006). A Cauchy or initial value problem is solved with the vertical coordinate $z$ playing the role of time, and using the photospheric boundary conditions as the initial conditions. In addition to the photospheric field components, their horizontal derivatives are also used. However, this initial value problem is mathematically ill-posed, since the solutions do not depend continuously on the photospheric initial values. Therefore, the methods proposed here involve regularization or smoothing schemes. The problem of ill-posedness also occurs for constant- $\alpha$ and current-free fields if these are determined, for instance, from the values of the vertical field component $B_{z}$ and its vertical derivative $\partial B_{z} / \partial z$ (which by use of Eq. (2) can be expressed in terms of the horizontal derivatives of the horizontal components $B_{x}$ and $B_{y}$ ) at the photosphere (Seehafer \& Staude 1983; Amari et al. 1998).

- Grad-Rubin methods (Grad \& Rubin 1958; Sakurai 1981; Amari et al. 1997, 1999, 2006; Wheatland 2004, 2006). An electric current-magnetic field iteration is applied with fieldaligned currents calculated according to $\boldsymbol{j}=\left(1 / \mu_{0}\right) \nabla \times \boldsymbol{B}=$ $\left(\alpha / \mu_{0}\right) \boldsymbol{B}$, and magnetic fields calculated by using the BioSavart law. The normal field component $B_{n}$ is prescribed on the whole boundary and $\alpha$ on a part of the boundary where $B_{n}$ is either positive or negative. A modification of the method for global fields was proposed by Aly \& Seehafer (1993), with the additional condition that the field be radial at an exterior source surface; this condition is usual in global potential-field extrapolations and simulates the effect of the solar wind. Convergence of the iteration and existence of the solution can be proven for sufficiently small $|\alpha|$ (Bineau 1972; Kaiser et al. 2000; Boulmezaoud \& Amari 2000). Such existence proofs are not available for the other methods listed here.
- Boundary-integral methods (Yan \& Sakurai 1997, 2000; Yan 2003; Yan \& Li 2006; He \& Wang 2006). The magnetic field is represented by an integral over the boundary of the considered volume involving an auxiliary function. The problem is solved iteratively whereby the auxiliary function has to be determined such that a remaining volume integral vanishes. The magnetic vector on the photospheric boundary is used as input.

- Relaxation methods (Yang et al. 1986; Mikic \& McClymont 1994; Roumeliotis 1996; Valori et al. 2005). The magnetohydrodynamic equations are simulated, generally in a simplified or modified form. The equation for the fluid velocity contains a viscous dissipation term but no external forcing terms, and the pressure term is neglected. If some energy inflow through the boundary is allowed, this must be exceeded by the dissipative losses. Asymptotically in time, a quiescent state with a force-free magnetic field is reached. Photospheric vector magnetograms are used as boundary data.

- Optimization methods (Wheatland et al. 2000; Wiegelmann 2004). A positive semidefinite functional $\mathcal{L}$ of the magnetic field is minimized using a prescription for evolving $\boldsymbol{B}$. If $\mathcal{L}=0$ is reached, $\nabla \cdot \boldsymbol{B}=0$ and $(\nabla \times \boldsymbol{B}) \times \boldsymbol{B}=\mathbf{0}$ are satisfied throughout the volume considered. Again, photospheric vector magnetograms are used as boundary data. An adaption of the method for the calculation of global force-free fields in spherical geometry was proposed by Wiegelmann (2007), while extensions to non-force-free fields, with pressure gradients and gravity included, were developed by Wiegelmann \& Inhester (2003) and Wiegelmann \& Neukirch (2006).

Discussions of the nonlinear extrapolation methods are found in Aly (1989), Sakurai (1989), Amari et al. (1997), McClymont et al. (1997) and Schrijver et al. (2006).

With the exception of the direct vertical integration schemes, the nonlinear extrapolation methods impose boundary conditions on the whole boundary of the volume in which the solution is calculated, in practice usually a rectangular box over the magnetogram area. For the Grad-Rubin methods, the corresponding boundary value problem is well posed (at least for sufficiently small $|\alpha|$ and perhaps up to the uniqueness of the solution). However, these methods do not exploit the full information content of vector magnetograms. The boundary-integral, relaxation and optimization methods prescribe the magnetic vector on the boundary. Vector magnetograms provide the boundary values for the lower, photospheric part of the boundary, while some assumption is made for the remaining parts, e.g. keeping the values of an initial potential field there fixed, so that altogether the full magnetic vector is prescribed on the whole closed boundary. This overdetermines the force-free field. Therefore, the calculations will lead to correct solutions only if the prescribed boundary values are consistent with the condition of force-freeness. This cannot be expected, however, for several reasons. First of all, the magnetic field is not force-free in the photosphere and in the lower chromosphere (possibly with the exception of sunspot areas, where the field is strongest). Furthermore, there are measurement errors, in particular in the transverse field components (perpendicular to the line of sight of the observer), that would destroy the compatibility of a magnetogram with the condition of force-freeness.

One way to alleviate these problems is a preprocessing of the magnetograph data as suggested by Wiegelmann et al. (2006). The vector components of the total magnetic force and the total magnetic torque on the volume considered are given by six 
boundary integrals that have to vanish if the magnetic field is force-free in the full volume (Molodenskii 1969; Aly 1984, 1989; Low 1985). The preprocessing changes the boundary values of $\boldsymbol{B}$ within the error margins of the measurement in such a way that the moduli of the six boundary integrals are minimized. The resulting boundary values are expected to be more suitable for an extrapolation into a force-free field than the original values.

In the practical calculations, the convergence properties of the used iterations or evolutions, as well as the calculated fields themselves, are very sensitive to small-scale noise and apparent discontinuities in the photospheric magnetograph data. This problem should, in principle, disappear if small spatial scales were sufficiently resolved. However, the numerical effort for that would be enormous. Also, the small-scale fluctuations in the magnetograms are presumed to affect the solutions only in a very thin boundary layer close to the photosphere. Therefore, a smoothing of the data is included in the preprocessing.

In this paper, we follow the suggestion of Wiegelmann et al. (2006) and expand their method of preprocessing photospheric vector magnetograms. The paper is organized as follows: in Sect. 2 we describe a new method for the preprocessing; then, in Sect. 3, we apply it to a known nonlinear force-free test field; finally, in Sect. 4, we draw conclusions and discuss our results.

\section{Procedure}

The strategy of preprocessing is to define a functional $L$ of the boundary values of $\boldsymbol{B}$, such that on minimizing $L$ the total magnetic force and the total magnetic torque on the considered volume, as well as a quantity measuring the degree of small-scale noise in the boundary data, are simultaneously made small. Each of the quantities to be made small is measured by an appropriately defined subfunctional included in $L$. The different subfunctionals are weighted in order to control their relative importance. Furthermore, domain borders are implemented so that the field is only allowed to change within these borders that are defined by the error margins of the magnetographic measurement.

\subsection{The objective quantities}

The total magnetic force on a volume $V$ is given by

$$
\begin{aligned}
\mathcal{F} & =\int_{V} \boldsymbol{j} \times \boldsymbol{B} \mathrm{d} V \\
& =\frac{1}{\mu_{0}} \int_{V}(\nabla \times \boldsymbol{B}) \times \boldsymbol{B} \mathrm{d} V \\
& =\int_{V} \operatorname{div} \mathrm{T} \mathrm{d} V \\
& =\oint_{\partial V} \mathrm{~T} \mathrm{~d} \boldsymbol{S},
\end{aligned}
$$

where $\partial V$ is the surface of $V$, and in the last step Gauss' theorem was used; $T$ is the magnetic stress tensor,

$\mathrm{T}_{i j}=-\frac{\boldsymbol{B}^{2}}{2 \mu_{0}} \delta_{i j}+\frac{1}{\mu_{0}} B_{i} B_{j}$,

and $\operatorname{div} \mathrm{T}$ its divergence,

$[\operatorname{div} \mathrm{T}]_{i}=\partial_{j} \mathrm{~T}_{i j}$

(we use the summation convention). Quite analogously, the total magnetic torque on $V$ can be written as

$\mathcal{N}=\int_{V} \boldsymbol{r} \times(\boldsymbol{j} \times \boldsymbol{B}) \mathrm{d} V=\int_{V} \operatorname{div} \hat{\mathrm{T}} \mathrm{d} V=\oint_{\partial V} \hat{\mathrm{T}} \mathrm{d} \boldsymbol{S}$ with

$\hat{\mathrm{T}}_{i j}=\varepsilon_{i k l} r_{k} \mathrm{~T}_{l j}$

If, as in our case, the magnetic field is force-free in $V$, the surface integrals in Eqs. (3) and (6) have to vanish. These integrals are over the whole boundary of $V$, but we approximate them by integrals just over the photospheric magnetogram area. This will only be justified if the net magnetic flux through the magnetogram area vanishes (Wiegelmann et al. 2006). Actually, $\int_{\partial V} \boldsymbol{B} \mathrm{d} \boldsymbol{S}=0, \mathcal{F}=\mathbf{0}$ and $\mathcal{N}=\mathbf{0}$ are fully independent conditions, and the vanishing of the volume integrals of higher-order moments of $\boldsymbol{j} \times \boldsymbol{B}$ would give additional independent conditions. Our intention is that all relevant magnetic flux is closed on the photosphere, and the field on the rest of the boundary is so weak that its contribution to the surface integrals for $\mathcal{F}$ and $\mathcal{N}$ is negligible.

With $S$ denoting the magnetogram area in the plane $r_{3}=0$, $\mathcal{F}$ and $\mathcal{N}$, given by Eqs. (3) and (6), are then approximated by

$\begin{array}{ll}\mathcal{F}=-\int_{S} \mathrm{Te}_{3} \mathrm{~d} r_{2} \mathrm{~d} r_{3} ; & \mathcal{F}_{i}=-\int_{S} \mathrm{~T}_{i 3} \mathrm{~d} r_{2} \mathrm{~d} r_{3}, \\ \mathcal{N}=-\int_{S} \hat{\mathrm{T}} \boldsymbol{e}_{3} \mathrm{~d} r_{2} \mathrm{~d} r_{3} ; & \mathcal{N}_{i}=-\int_{S} \hat{\mathrm{T}}_{i 3} \mathrm{~d} r_{2} \mathrm{~d} r_{3},\end{array}$

where $\boldsymbol{e}_{3}$ is the unit vector in the positive $r_{3}$ direction, pointing upward in the atmosphere. Writing $x, y, z$ for the components of $\boldsymbol{r}$, the condition of force-freeness reads

$$
\begin{aligned}
& \mu_{0} \mathcal{F}_{x}=-\int_{S} B_{x} B_{z} \mathrm{~d} x \mathrm{~d} y=0 \\
& \mu_{0} \mathcal{F}_{y}=-\int_{S} B_{y} B_{z} \mathrm{~d} x \mathrm{~d} y=0 \\
& 2 \mu_{0} \mathcal{F}_{z}=\int_{S}\left(B_{x}^{2}+B_{y}^{2}-B_{z}^{2}\right) \mathrm{d} x \mathrm{~d} y=0,
\end{aligned}
$$

while the condition of torque-freeness is expressed by

$$
\begin{aligned}
& 2 \mu_{0} \mathcal{N}_{x}=\int_{S} y\left(B_{x}^{2}+B_{y}^{2}-B_{z}^{2}\right) \mathrm{d} x \mathrm{~d} y=0 \\
& 2 \mu_{0} \mathcal{N}_{y}=\int_{S} x\left(-B_{x}^{2}-B_{y}^{2}+B_{z}^{2}\right) \mathrm{d} x \mathrm{~d} y=0 \\
& \mu_{0} \mathcal{N}_{z}=\int_{S}\left(y B_{x} B_{z}-x B_{y} B_{z}\right) \mathrm{d} x \mathrm{~d} y=0
\end{aligned}
$$

(see Molodenskii 1969; Aly 1989). The better these integral conditions are fulfilled, the higher is the expected compatibility of the boundary data with the requirement that the magnetic field be force-free in the volume $V$. However, due to their integral character and their finite number, the conditions are only necessary, not sufficient.

Another task is to decrease the amount of small-scale irregularities in the data, which we call smoothing. One possibility here is to calculate the horizontal second-order derivatives of the photospheric field, combined in the two-dimensional Laplacian $\Delta_{x y}=\partial^{2} / \partial x^{2}+\partial^{2} / \partial y^{2}$. The square of $\Delta_{x y} \boldsymbol{B}(x, y, z=0)$ can be used to estimate the smoothness of the photospheric boundary data because the second-order derivatives measure the curvature of the surfaces $B_{x}(x, y, z=0), B_{y}(x, y, z=0)$ and $B_{z}(x, y, z=0)$. 
Minimizing the integral of $\left[\Delta_{x y} \boldsymbol{B}(x, y, z=0)\right]^{2}$ over the magnetogram area $S$ is one method of averaging the field. The disadvantage here is that, if the modulus of $\Delta_{x y} \boldsymbol{B}(x, y, z=0)$ is decreased to zero on $S$, all global or local maxima or minima of the three field components on $S$ are removed, including the physically significant ones, and the total energy content of the field may be strongly reduced. Therefore, this method of smoothing can only be applied with great care.

A different method of smoothing a vector magnetogram, which we prefer here, is the windowed median (see, e.g., Press et al. 1989). In this method one chooses a small window around a given point, calculates the median of the field values in the window, and then minimizes the difference between the field value at the point and the median of the window. Calculating the median is a more stable way of averaging than the normal mean, which corresponds to minimizing the Laplacian, because it is much less influenced by strong disturbances in a small number of points. After several iterations the method will stabilize the changes; this way, the structures of the measurement are conserved even if the quantity minimized here is decreased to zero.

\subsection{The functional}

In discretized form, the squares of the total magnetic force and the total magnetic torque are given by

$$
\begin{aligned}
L_{1}= & \left(\sum_{P} B_{x} B_{z}\right)^{2}+\left(\sum_{P} B_{y} B_{z}\right)^{2}+\left(\sum_{P}\left(B_{x}^{2}+B_{y}^{2}-B_{z}^{2}\right)\right)^{2}, \\
L_{2}= & \left(\sum_{P} x\left(B_{x}^{2}+B_{y}^{2}-B_{z}^{2}\right)\right)^{2}+\left(\sum_{P} y\left(B_{x}^{2}+B_{y}^{2}-B_{z}^{2}\right)\right)^{2} \\
& +\left(\sum_{P} y B_{x} B_{z}-x B_{y} B_{z}\right)^{2},
\end{aligned}
$$

where the summation is over the points $P$ of a photospheric grid. Since there is no obvious reason to weight $L_{1}$ and $L_{2}$ differently, they are combined to

$L_{12}=L_{1}+L_{2}$.

This can only be done if the two quantities have been made dimensionless beforehand. We normalize $L_{1}$ to

$N_{L_{1}}=\left[\sum_{P}\left(\boldsymbol{B}^{(0)}\right)^{2}\right]^{2}=B_{\text {mean }}^{4} N^{2}$

where $\boldsymbol{B}^{(0)}$ is the original, magnetographically measured photospheric field, $B_{\text {mean }}$ the mean value of $\left|\boldsymbol{B}^{(0)}\right|$ over the magnetogram, and $N$ the number of photospheric grid points, and we normalize $L_{2}$ to

$N_{L_{2}}=\left[\sum_{P} \sqrt{x^{2}+y^{2}}\left(\boldsymbol{B}^{(0)}\right)^{2}\right]^{2}=h^{2}\left[\sum_{k=1}^{N_{x}} \sum_{l=1}^{N_{y}} \sqrt{k^{2}+l^{2}}\left(\boldsymbol{B}_{k l}^{(0)}\right)^{2}\right]^{2}$,

with $N_{x}$ and $N_{y}$ denoting the numbers of grid points in the $x$ and $y$ directions $\left(N_{x} \cdot N_{y}=N\right), h$ the grid spacing (assumed to be equal in the $x$ and $y$ directions) and $\boldsymbol{B}_{k l}^{(0)}$ the elements of the magnetogram matrix. With these normalizations, $L_{1}$ and $L_{2}$ are independent of the units of length and magnetic field and both are $\sim 1$ for a field that is neither force-free nor torque-free.
The smoothing functional is defined as ${ }^{1}$

$$
\begin{aligned}
L_{4}= & \sum_{i=x, y, z} \sum_{P}\left\{M _ { n } \left[B_{i}(x-n \cdot h, y-n \cdot h), \ldots\right.\right. \\
& \left.\left.\ldots, B_{i}(x+n \cdot h, y+n \cdot h)\right]-B_{i}(x, y)\right\}^{2},
\end{aligned}
$$

where $n$ is a positive integer number and $M_{n}$ the median of a rectangular window with $(2 n+1) \cdot(2 n+1)$ grid points centered about the point $(x, y)$. The values at the boundary of the magnetogram, where the method does not work, are left unchanged; these values are expected to be small in general. Also, in the practical extrapolations an artificial margin where the field vanishes is very often added to the magnetogram. $L_{4}$ is normalized to

$$
\begin{aligned}
N_{L_{4}}= & \sum_{i=x, y, z} \sum_{P}\left\{M _ { n } \left[B_{i}^{(0)}(x-n \cdot h, y-n \cdot h), \ldots\right.\right. \\
& \left.\left.\ldots, B_{i}^{(0)}(x+n \cdot h, y+n \cdot h)\right]+B_{i}^{(0)}(x, y)\right\}^{2},
\end{aligned}
$$

so that $L_{4} \sim 1$ for a field of maximum roughness.

Since just the relative weighting of the different subfunctionals is important for the minimization, the weighting of one of them can be set as equal to unity. We choose the form

$L=L_{12}+\mu_{4} \cdot L_{4}$

for the total functional to minimize. Like the number $n$ determining the window size for the smoothing, the weighting factor $\mu_{4}$ is yet undetermined.

\subsection{Minimization algorithm}

Minimizing a simple functional with just one minimum can be easily done by fast methods like Newton-Raphson iteration, but here we have a more complex functional and, to our knowledge, there is no way of knowing how many local minima exist or if there actually exists a global minimum. The success of the Newton-Raphson method strongly depends on the initial field for the iteration, since it just finds the nearest local minimum.

We use here the method of simulated annealing (see, e.g., Press et al. 1989; Rutenbar 1989; Corana et al. 1987; Siarry et al. 1997), which also finds the nearest local minimum, but opens a chance to leave it and to find a deeper one, within given domain borders. Simulated annealing works as follows: start with a given field and change the values of the vector components at one grid point randomly within a small interval (for example $0.5 \%$ around the initial values). Then calculate the functional that has to be minimized both for the initial and the changed field. If the new value of the functional, $L_{\text {new }}$, is smaller than the old one, $L_{\text {old }}$, then take the new field. If the new value is larger than the old one, then take the new field with a probability $\propto \exp \left\{-\frac{L_{\text {new }}-L_{\text {old }}}{T}\right\}$, where the "temperature" $T$ is used to control the behaviour of the algorithm. A sweep through the whole magnetogram is one iteration. Use the chosen field as the initial field for the next iteration. Start the algorithm with small values of $T$; this way, only smaller values of $L$ will be accepted. After some iterations a first minimum is reached. Increase the value of $T$; this way one opens a chance of leaving the first minimum. If the minimum has been left, decrease $T$ again and the algorithm will again find the nearest local minimum. In this way, the whole domain, as defined by the domain borders, will be scanned (changes to field values outside the domain borders defined before are not allowed).

\footnotetext{
1 In the nomenclature for the different subfunctionals of $L$ we follow Wiegelmann et al. (2006). Their subfunctional $L_{3}$ is not used by our method.
} 


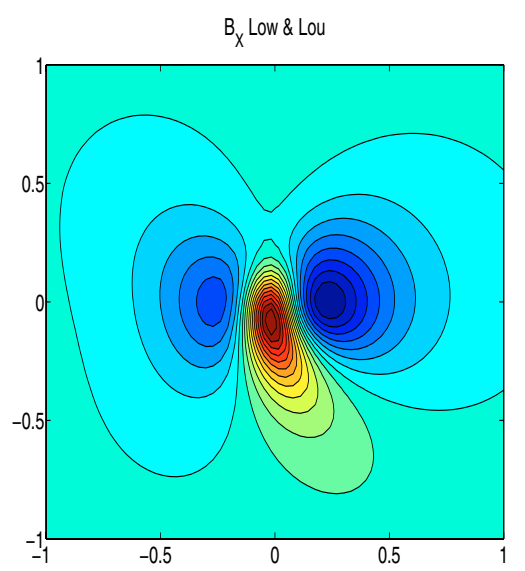

$B_{x}$ noisy

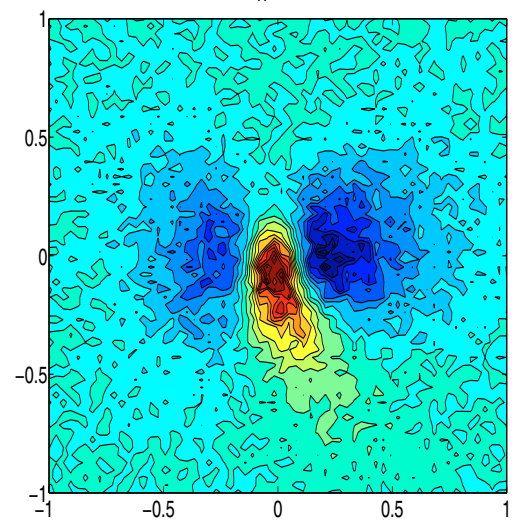

$B_{x}$ preprocessed

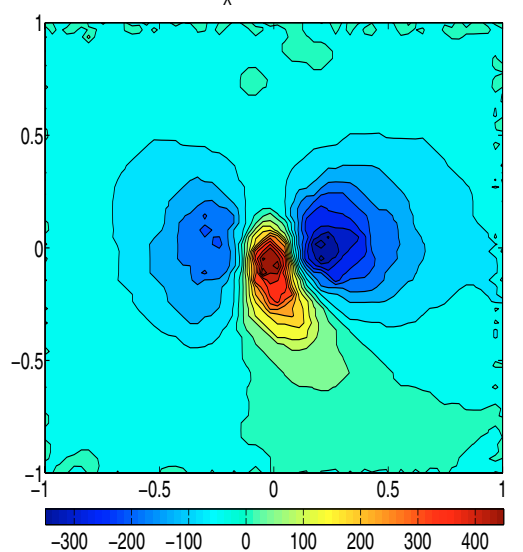

B Low \& Lou

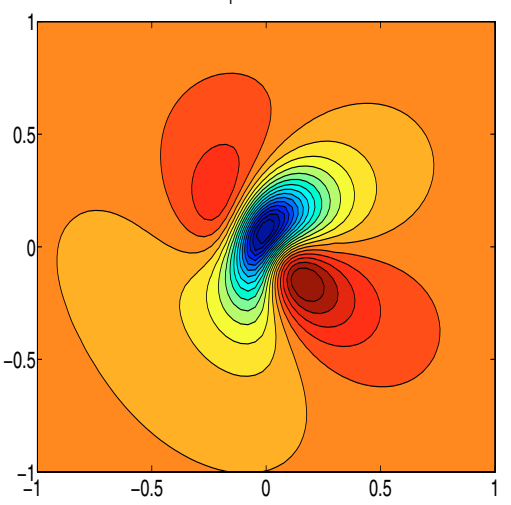

B noisy

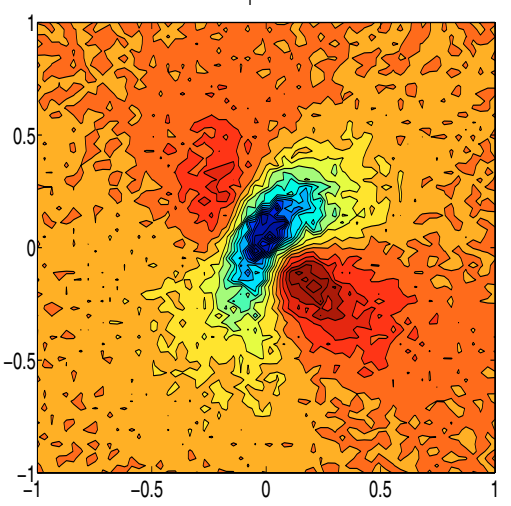

$B_{Y}$ preprocessed

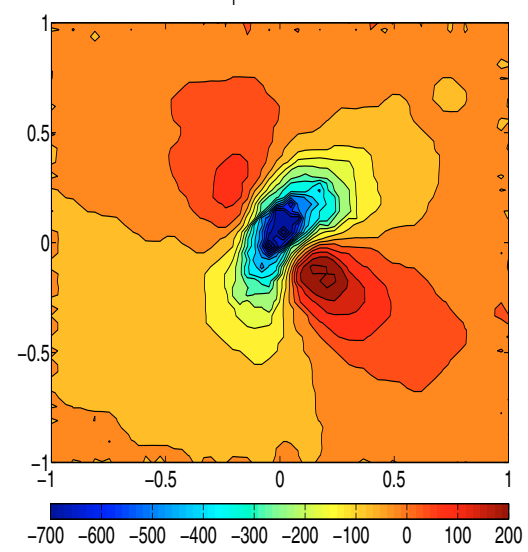

$B_{z}$ Low \& Lou

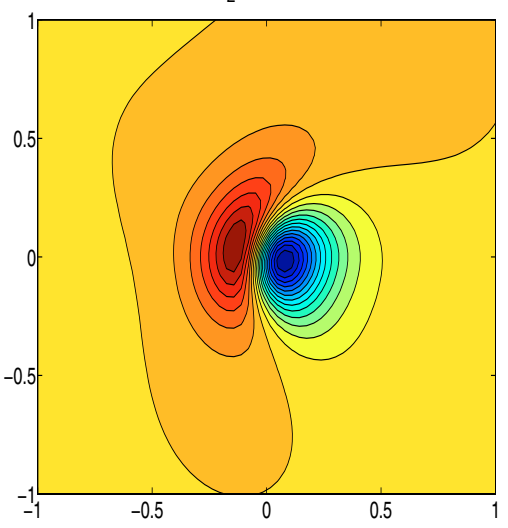

$B_{z}$ noisy

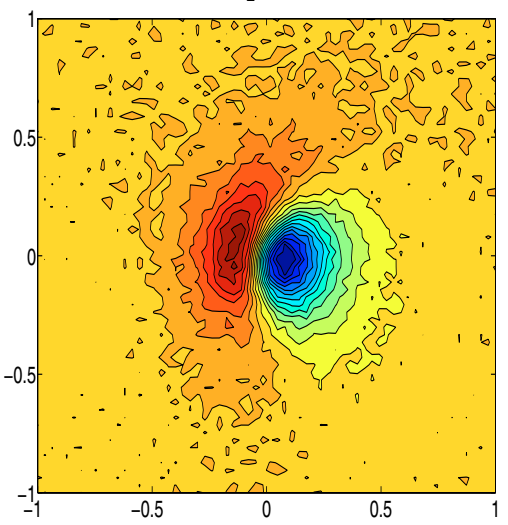

$B_{Z}$ preprocessed

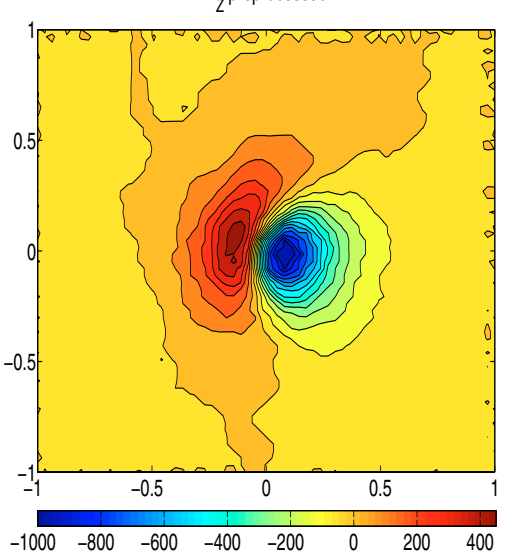

Fig. 1. Top row: magnetogram derived from the Low \& Lou test field. From left to right the three components $B_{x}, B_{y}, B_{z}$ are shown. Middle row: the same magnetogram as in the first row, but with noise added (for details see the main text). Bottom row: magnetogram resulting from preprocessing of the disturbed magnetogram shown in the second row.

\section{Application to the Low and Lou test field}

In order to test our preprocessing technique, we used one of the semi-analytical solutions found by Low \& Lou (1990). These have become standard test cases for extrapolations of nonlinear force-free magnetic fields (cf. Wheatland et al. 2000; Wiegelmann 2004; Wiegelmann et al. 2006; Amari et al. 2006; Schrijver et al. 2006; Valori et al. 2007). We chose a frequently used special solution, characterized by the parameters $n=1$, $m=1, l=0.3$ and $\Phi=\pi / 4$; for details see Low \& Lou (1990). The field was given in the cubic volume $-1 \leq x \leq 1$,
$-1 \leq y \leq 1,0 \leq z \leq 2$, so the test field is identical to FF1 in Amari et al. (2006) and Case I in Schrijver et al. (2006) and Valori et al. (2007). We used a numerical resolution of $64^{3}$ grid points, and the unit for the magnetic field was chosen such that the maximum strength of the photospheric normal component is $1000 \mathrm{G}$.

\subsection{Contours of the magnetogram}

In the top row of Fig. 1, from left to right the three components $B_{x}, B_{y}, B_{z}$ of a vector magnetogram derived from the 

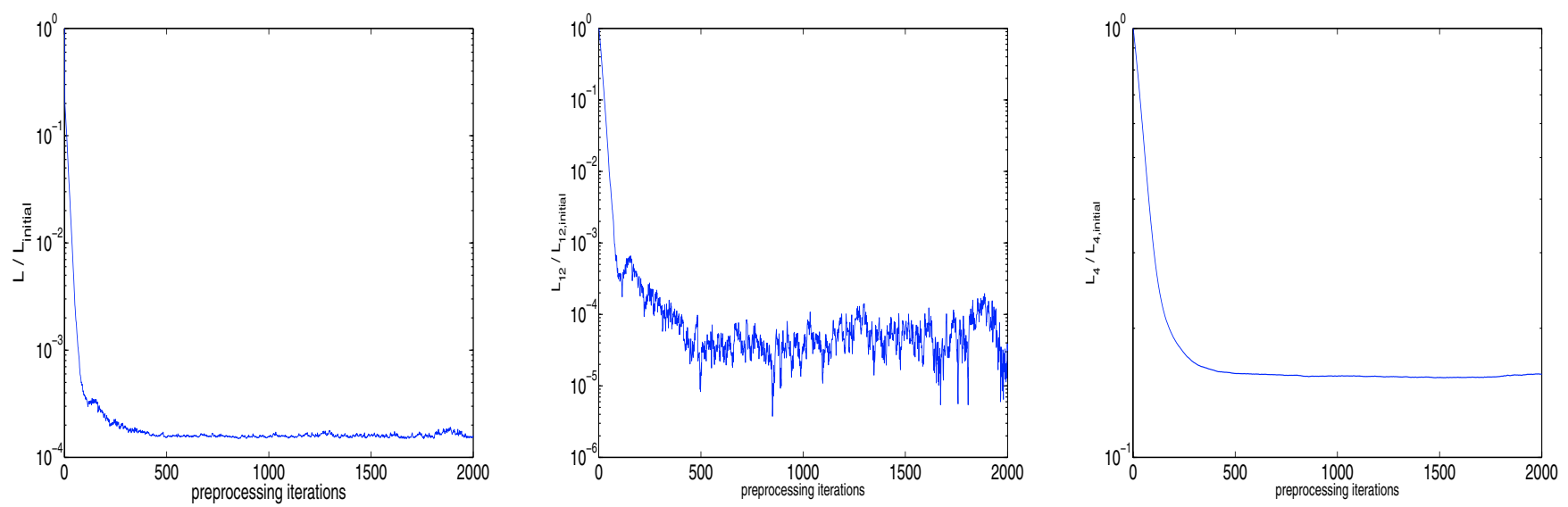

Fig. 2. Evolution of the total functional $L$ (left) and of the subfunctionals $L_{12}$ (center) and $L_{4}$ (right) in the course of the minimization on a logarithmic scale. All three quantities are normalized to their initial values.
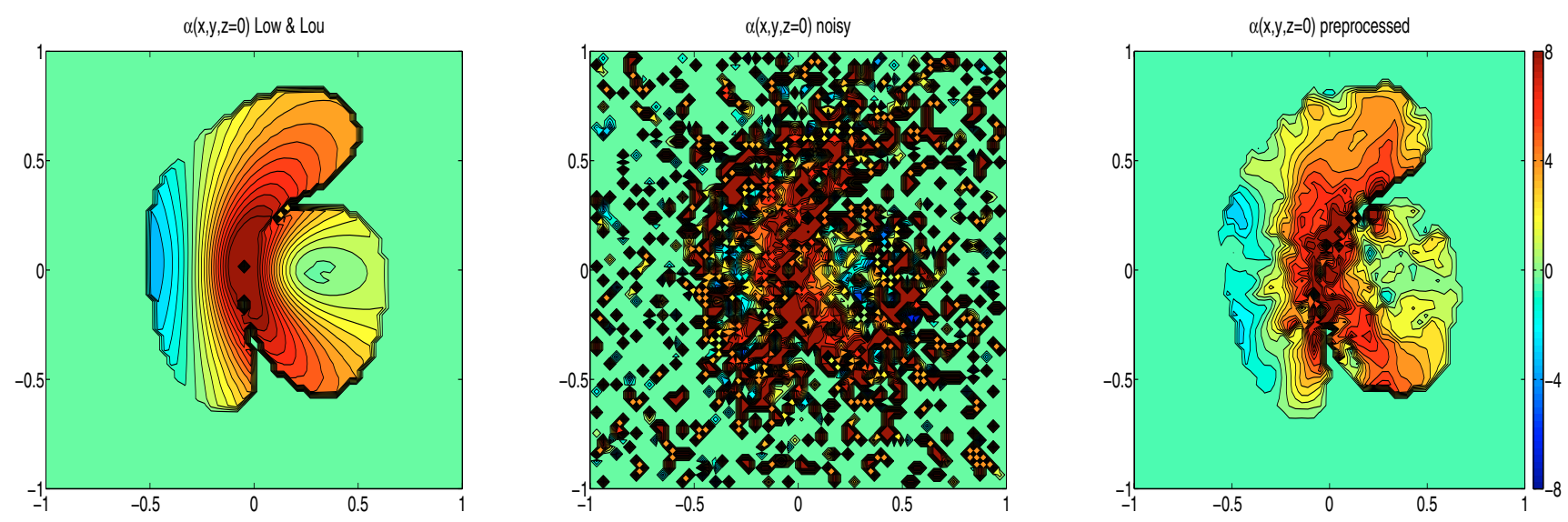

Fig. 3. Contours of the function $\alpha(x, y, z=0)$ for the undisturbed magnetogram (left; magnetogram shown in top row of Fig. 1), the disturbed magnetogram (center; magnetogram shown in middle row of Fig. 1) and the preprocessed magnetogram (right; magnetogram shown in bottom row of Fig. 1).

Low \& Lou test field are shown. The middle row shows the same magnetogram, but now with artificial noise. At every grid point of the magnetogram white noise was added, based on a relative error of $30 \%$ in the horizontal field components and $5 \%$ in the normal component, corresponding to the fact that the measurement of the transverse field components is much less accurate than that of the line-of-sight component (we consider a case where the $z$ direction coincides with the line of sight). Additionally, we assumed a field-independent error component (also white noise) with an absolute (maximum) amplitude of $35 \mathrm{G}$ in all three field components. Starting from the disturbed magnetogram we applied our preprocessing as described in Sect. 2. The smoothing was weighted with a factor of $\mu_{4}=0.1$ in the functional $L$ (see Sect. 3.4 for a discussion of how to find an optimal value for $\mu_{4}$ ) and carried out using the windowed median method with $n=1$. The domain borders for the minimization by the method of simulated annealing were chosen to be $85 \mathrm{G}$ for the transverse components and $35 \mathrm{G}$ for the normal component.

In the bottom row of Fig. 1 the preprocessed magnetogram is shown. Comparison with the original undisturbed magnetogram (top row of the figure) shows that the method works remarkably well. The neutral lines where the respective field components change sign, as well as the poles (minima and maxima), are mainly recovered. Also, the small-scale noise in the disturbed magnetogram (middle row of the figure) has been removed.
In Fig. 2 the evolutions of $L$ and of the two subfunctionals $L_{12}$ and $L_{4}$ (cf. Sect. 2) in the course of the minimization are shown. All three quantities are normalized to their initial values in order to show, in particular, the decrease related to the initial values. As one can see, after 100 iterations the algorithm first found a local minimum of $L$, but then it drove the system out of this minimum to find one where, in particular, $L_{12}$ is smaller by an order of magnitude.

\subsection{The function $\alpha(x, y, z=0)$}

The function $\alpha(\boldsymbol{r})$ defined by Eq. (1) contains much information on the topology of the magnetic field lines since these lie in the surfaces $\alpha=$ constant. In particular, the values of $\alpha$ at any two points in the photosphere that are connected by a field line above the photosphere must be identical. For the level of the photosphere, $\alpha$ can be calculated from the magnetogram according to

$\alpha(x, y, z=0)=\frac{\partial_{x} B_{y}(x, y, z=0)-\partial_{y} B_{x}(x, y, z=0)}{B_{z}(x, y, z=0)}$.

Obviously, since one has to combine three noisy quantities and, moreover, to differentiate two of them, $\alpha(x, y, z=0)$ is more sensitive to noise in the magnetographic measurement than the field components themselves. In Fig. 3, from left to right the function $\alpha(x, y, z=0)$ is shown as calculated from the undisturbed 
magnetogram derived from the Low \& Lou field, the disturbed magnetogram and the preprocessed magnetogram (cf. Sect. 3.1). The noise overshadows the signal of $\alpha$ strongly. However, the preprocessing is even able to recover the main structures of this heavily disturbed function.

\subsection{Figures of merit for extrapolated fields}

A critical test of the effect of the preprocessing is to extrapolate from disturbed photospheric vector magnetograms before and after the preprocessing, and compare the results with those of extrapolations starting from the exact photospheric values of the Low \& Lou test solution, as well as with the exact field values of this solution in the volume above the photosphere. We used the magneto-frictional relaxation method of Valori et al. (2005) (see also Valori et al. 2007) for corresponding extrapolations.

To assess the quality of extrapolations, Schrijver et al. (2006) developed a number of figures of merit (hereafter FoM). The FoM are measures of the discrepancy between an exactly known test field, $\boldsymbol{B}$, and the extrapolated field, $\boldsymbol{b}$, namely

$$
\begin{aligned}
& C_{\mathrm{vec}}=\frac{\sum_{i} \boldsymbol{B}_{i} \cdot \boldsymbol{b}_{i}}{\left(\sum\left|\boldsymbol{B}_{i}\right|^{2} \sum_{i}\left|\boldsymbol{b}_{i}\right|^{2}\right)^{1 / 2}}, \\
& C_{\mathrm{CS}}=\frac{1}{M} \sum_{i} \frac{\boldsymbol{B}_{i} \cdot \boldsymbol{b}_{i}}{\left|\boldsymbol{B}_{i}\right|\left|\boldsymbol{b}_{i}\right|}, \\
& E_{\mathrm{n}}=\frac{\sum_{i}\left|\boldsymbol{b}_{i}-\boldsymbol{B}_{i}\right|}{\sum_{i}\left|\boldsymbol{B}_{i}\right|} ; \quad E_{\mathrm{n}}^{\prime}=1-E_{\mathrm{n}}, \\
& E_{\mathrm{m}}=\frac{1}{M} \sum_{i} \frac{\left|\boldsymbol{b}_{i}-\boldsymbol{B}_{i}\right|}{\left|\boldsymbol{B}_{i}\right|} ; \quad E_{\mathrm{m}}^{\prime}=1-E_{\mathrm{m}}, \\
& \varepsilon=\frac{\sum_{i}\left|\boldsymbol{b}_{i}\right|^{2}}{\sum_{i}\left|\boldsymbol{B}_{i}\right|^{2}},
\end{aligned}
$$

where $M$ is the number of grid points in the considered threedimensional domain and the summation is over these grid points. $C_{\text {vec }}$ is the scalar product between the unit vectors corresponding to $\boldsymbol{B}$ and $\boldsymbol{b}$, respectively, in the $3 M$-dimensional space spanned by the field values of the whole grid, and $C_{\mathrm{CS}}$ is the mean value over the grid of the scalar products between the unit vectors of the two fields in three-dimensional space. $E_{\mathrm{n}}$ and $E_{\mathrm{m}}$ measure the vector error $|\boldsymbol{B}-\boldsymbol{b}|$ with a global and a local normalization, respectively, and $\varepsilon$ is the ratio between the magnetic energies of the extrapolated and exact fields.

In Fig. 4, the FoM are shown for three-dimensional fields extrapolated from a magnetogram containing the exact field values of the Low \& Lou solution, a noisy magnetogram obtained from this as described in Sect. 3.1, and a magnetogram obtained by applying our preprocessing procedure to the noisy magnetogram. Instead of $E_{\mathrm{n}}$ and $E_{\mathrm{m}}, E_{\mathrm{n}}^{\prime}$ (see Eq. (27)) and $E_{\mathrm{m}}^{\prime}$ (see Eq. (28)) are shown, so that all displayed measures are equal to unity in the case of a full match. Naturally, the extrapolation of the original magnetogram reproduces the exact field best. The agreement is markedly worse for the extrapolation of the noisy magnetogram, but is again significantly improved for the extrapolation using the preprocessed magnetogram. Only the energy ratio $\varepsilon$ shows a deviating behaviour. The reduced energy content of the field extrapolated from the preprocessed magetogram compared to that extrapolated from the noisy magnetogram can be explained by the smoothing procedure, which removes a part of the energy in the small spatial scales. That the noisy magnetogram gives a higher energy content of the field than the undisturbed one seems to be mainly a result of our addition of a noise component with

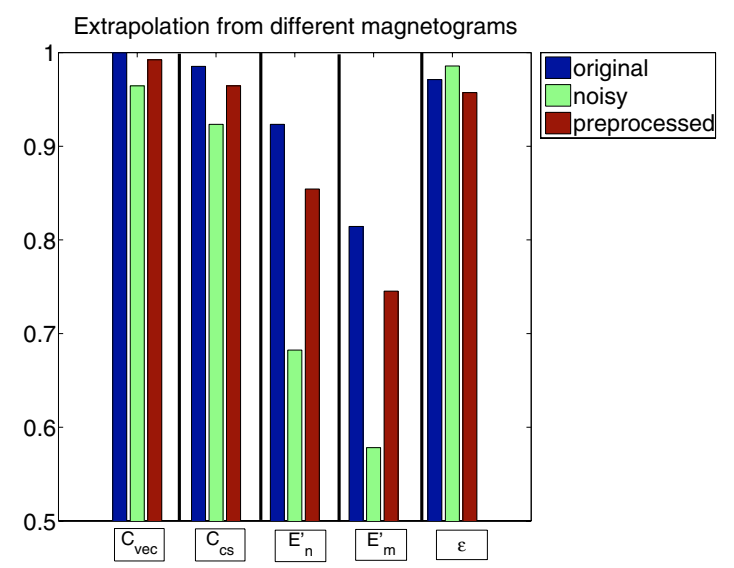

Fig. 4. Figures of merit $C_{\mathrm{vec}}, C_{\mathrm{CS}}, E_{\mathrm{n}}^{\prime}, E_{\mathrm{m}}^{\prime}$ and $\varepsilon$ (from left to right) for three-dimensional fields extrapolated from the original magnetogram with the exact field values of the Low \& Lou solution (blue; magnetogram shown in top row of Fig. 1), the disturbed magnetogram (green; magnetogram shown in middle row of Fig. 1) and the preprocessed magnetogram (red; magnetogram shown in bottom row of Fig. 1).

field-independent amplitude (cf. Sect. 3.1), which increases, in particular, the energy in the weak-field regions.

\subsection{Searching for an optimal $\mu_{4}$}

The expression for the minimization functional $L$, given by Eq. (23), contains the free parameter $\mu_{4}$. If $\mu_{4}=0$, the subfunctional $L_{12}$, measuring the deviation from force-freeness and torque-freeness, is minimized very effectively. For our noisy test magnetogram generated from the Low \& Lou solution and with the chosen domain borders for the minimization, the value of the subfunctional $L_{12}$ is decreased to zero (within machine accuracy) relatively quickly. For $\mu_{4} \neq 0, L_{12}=0$ is no longer reached. This results from a competition between two different minimization objectives. Increasing $\mu_{4}$ gives $L_{4}$ a higher weight and, thus, enforces the smoothing and inhibits the decrease of $L_{12}$. On the other hand, decreasing $\mu_{4}$ gives $L_{12}$ the possibility to reach values close to zero, but inhibits the smoothing. So $L_{12}$ and $L_{4}$ are competitive functionals, and finding the right $\mu_{4}$ is an optimization problem on its own.

Unfortunately, there is no simple way to determine an optimum $\mu_{4}$. One could extrapolate from magnetograms derived from a test field and compare the extrapolated fields with the exact solution. However, the numerical effort for this would be very high and the result would only be valid for the test field.

Since $L$ (see Eq. (23)) depends on $\mu_{4}$, it seems better to look at a different functional that is independent of $\mu_{4}$ to investigate the influence of different weightings on the minimization process. The simplest choice for such a functional is the sum $L_{12}+L_{4}$. It is then, in principle, possible to find an optimal $\mu_{4}$ by the method of simulated annealing. Thus, one can minimize $L$ for different values of $\mu_{4}$ and then compare the values of $L_{12}+L_{4}$ for the associated photospheric magnetic fields that minimize $L$, i.e., one compares the sums $L_{12}^{(\mathrm{final})}\left(\mu_{4}\right)+L_{4}^{(\mathrm{final})}\left(\mu_{4}\right)$ of the final values of $L_{12}$ and $L_{4}$ reached by the minimization of $L$ for different values of $\mu_{4}$. At the same time, in view of the arbitrariness of the choice $L_{12}+L_{4}$, the $\mu_{4}$ dependence of the final values of the two individual subfunctionals $L_{12}$ and $L_{4}$ should be considered.

In Fig. 5, the dependence of the final values of $L_{12}, L_{4}$ and $L_{12}+L_{4}$ on $\mu_{4}$ is shown. The calculations were done using the noisy magnetogram generated from the Low \& Lou test solution 


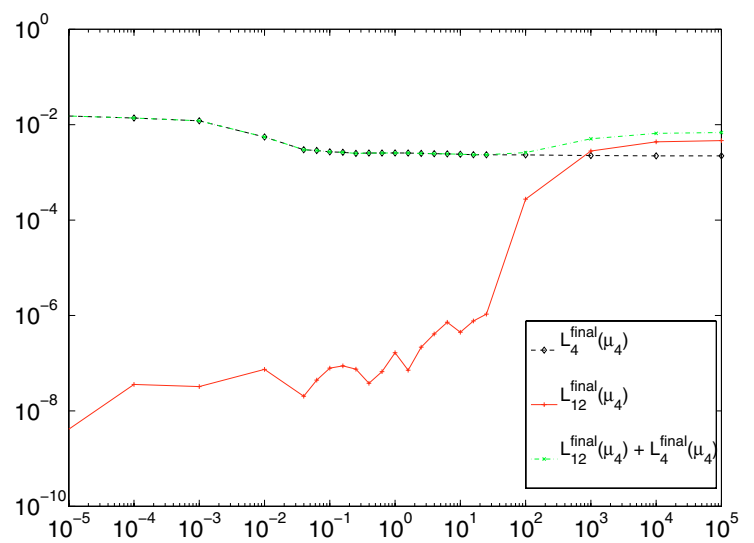

Fig. 5. Dependence of the final values of $L_{12}, L_{4}$ and $L_{12}+L_{4}$ on the parameter $\mu_{4}$ on a doubly logarithmic scale. The values were calculated by applying the minimization procedure for $L$ to the noisy magnetogram (shown in middle row of Fig. 1) generated from the Lou \& Lou test solution.

(cf. Sect. 3.1). One can see that $L_{12}^{(\mathrm{final})}\left(\mu_{4}\right)+L_{4}^{(\mathrm{final})}\left(\mu_{4}\right)$ reaches a broad minimum in the interval $0.1 \lessgtr \mu_{4} \lesssim 10$. We have chosen $\mu_{4}=0.1$, a value at the left border of this interval, for our calculations (cf. Sect. 3.1), in doing this a low final value of $L_{12}$ is found and the final value of $L_{4}$ has reached a saturation, decreasing only slightly for further increased values of $\mu_{4}$.

\section{Conclusions}

We have developed a new method for the preprocessing of solar photospheric vector magnetograms that makes them more suitable for extrapolations into three-dimensional nonlinear forcefree magnetic fields in the chromosphere and corona. The principle of the method is the one suggested by Wiegelmann et al. (2006), namely, to minimize a functional, $L$, of the photospheric field values whereby the total magnetic force and the total magnetic torque on the considered volume above the photosphere, as well as a quantity measuring the degree of small-scale noise in the photospheric boundary data, are simultaneously made small.

For the minimization we use the method of simulated annealing. Compared to methods like Newton-Raphson iteration, this method has the advantage of finding not only the local minimum closest to the chosen initial field, but also of absolutely minimizing the functional $L$. The smoothing of noisy magnetograph data is performed by windowed median averaging. Compared to other methods in use, this method of averaging better conserves the real structures in the measurements.

We have applied our method of preprocessing to a magnetogram derived from one of the semi-analytical nonlinear force-free magnetic fields devised by Low \& Lou (1990). The magnetogram was exposed to an artificial noise. The algorithm recovered all main structures of the magnetogram and removed small-scale noise. Even quantities that are very sensitive to noise, like the function $\alpha(\boldsymbol{r})$ in the plane of the magnetogram, could be reproduced very well. The main test of the effect of the preprocessing was to extrapolate from the noisy photospheric vector magnetogram before and after the preprocessing, and compare the results with those of extrapolations starting from the exact photospheric values of the Low \& Lou test solution, as well as with the exact field values of this solution itself. To assess the quality of the extrapolations, the figures of merit developed by Schrijver et al. (2006) were used. The preprocessing was found to significantly improve the agreement of the extrapolated field with the exact field.

The method of minimization by simulated annealing is easy to adjust to special requirements, such as changed or new minimization objectives, since the functional $L$ is treated directly, i.e., only the values of the functional itself have to be calculated. In contrast with, e.g., Newton-Raphson iteration, derivatives of $L$ are not needed.

Our minimization functional contains a free weighting parameter, $\mu_{4}$, that controls the relative influence of the requirements of force-freeness and torque-freeness on the one hand, and smoothness of the photospheric field on the other hand. We have outlined a way to find an optimal value of $\mu_{4}$.

The original motivation for the preprocessing was to make vector magnetograph data more suitable for extrapolation methods that prescribe the full magnetic vector on the whole closed boundary of the considered volume, such as the relaxation and optimization methods, since prescribing the full vector overdetermines the force-free field. Making the the magnetograms more compatible with the condition of force-freeness can also be useful for extrapolations with Grad-Rubin methods since, e.g., the values of the function $\alpha(\boldsymbol{r})$, which are determined from the magnetogram on a part of the photospheric boundary, must be identical at the photospheric end points of a field line. Moreover, the determination of $\alpha(\boldsymbol{r})$ is very sensitive to noise, so that a smoothing of the data, as done by our method, also appears advisable. Finally, smoothing of the magnetograms is an indispensable part of extrapolations by direct vertical integration, since small-scale errors can grow exponentially with height. Thus, our method of smoothing could also be useful for this kind of extrapolation.

Acknowledgements. This work was supported by the Deutsche Forschungsgemeinschaft (DFG) through grants HO 1424/9-1 and SE 662/11-1.

\section{References}

Alissandrakis, C. E. 1981, A\&A, 100, 197

Altschuler, M. D., \& Newkirk, Jr., G. 1969, Sol. Phys., 9, 131

Aly, J. J. 1984, ApJ, 238, 349

Aly, J. J. 1989, Sol. Phys., 120, 19

Aly, J. J., \& Seehafer, N. 1993, Sol. Phys., 144, 243

Amari, T., Aly, J. J., Luciani, J. F., Boulmezaoud, T. Z., \& Mikic, Z. 1997, Sol. Phys., 174, 129

Amari, T., Boulmezaoud, T. Z., \& Maday, Y. 1998, A\&A, 339, 252

Amari, T., Boulmezaoud, T. Z., \& Mikic, Z. 1999, A\&A, 350, 1051

Amari, T., Boulmezaoud, T. Z., \& Aly, J. J. 2006, A\&A, 446, 691

Bineau, M. 1972, Comm. Pure Appl. Math., 25, 77

Boulmezaoud, T. Z., \& Amari, T. 2000, Z. angew. Math. Phys., 51, 942

Bungey, T. N., Titov, V. S., \& Priest, E. R. 1996, A\&A, 308, 233

Chiu, Y. T., \& Hilton, H. H. 1977, ApJ, 212, 873

Corana, A., Marchesi, M., Martini, C., \& Ridella, S. 1987, ACM Transactions on Mathematical Software (TOMS), 13, 262

Cuperman, S., Ofman, L., \& Semel, M. 1990, A\&A, 230, 193

Démoulin, P., Cuperman, S., \& Semel, M. 1992, A\&A, 263, 351

Gary, G. A. 1989, ApJS, 69, 323

Gary, G. A. 2001, Sol. Phys., 203, 71

Grad, H., \& Rubin, H. 1958, in Proc. 2nd Int. Conf. Peaceful Uses of Atomic Energy (Geneva: United Nations), 31, 190

He, H., \& Wang, H. 2006, MNRAS, 369, 207

Kaiser, R., Neudert, M., \& von Wahl, W. 2000, Commun. Math. Phys., 211, 111 Low, B. C. 1985, in Measurements of Solar Vector Magnetic Fields, ed. M. J. Hagyard, NASA Conf. Publ., 2374, 49

Low, B. C., \& Lou, Y. Q. 1990, ApJ, 352, 343

McClymont, A. N., Jiao, L., \& Mikić, Z. 1997, Sol. Phys., 174, 191

Mikic, Z., \& McClymont, A. N. 1994, in Solar Active Region Evolution:

Comparing Models with Observations, ed. K. S. Balasubramaniam, \& G. W.

Simon (San Francisco: ASP), ASP Conf. Ser., 68, 225

Molodenskii, M. M. 1969, Soviet Ast., 12, 585

Nakagawa, Y., \& Raadu, M. A. 1972, Sol. Phys., 25, 127 
Press, W. H., Flannery, B. P., Teukolsky, S. A., \& Vetterling, W. T. 1989, Numerical Recipes, The Art of Scientific Computing, FORTRAN Version (Cambridge, England: Cambridge University Press)

Roumeliotis, G. 1996, ApJ, 473, 1095

Rutenbar, R. A. 1989, Circuits and Devices Magazine, IEEE, 5, 19

Sakurai, T. 1981, Sol. Phys., 69, 343

Sakurai, T. 1989, Space Sci. Rev., 51, 11

Schatten, K. H., Wilcox, J. M., \& Ness, N. F. 1969, Sol. Phys., 6, 442

Schmidt, H. U. 1964, in AAS/NASA Symposium on the Physics of Solar Flares, ed. W. N. Hess, NASA SP-50 (Washington, D.C.: NASA), 107

Schrijver, C. J., DeRosa, M. L., Metcalf, T. R., et al. 2006, Sol. Phys., 235, 161

Seehafer, N. 1978, Sol. Phys., 58, 215

Seehafer, N. 1986, Sol. Phys., 105, 223

Seehafer, N. 1990, Sol. Phys., 125, 219

Seehafer, N., \& Staude, J. 1983, Phys. Solariterr., 22, 5

Semel, M. 1988, A\&A, 198, 293

Siarry, P., Berthiau, G., Durdin, F., \& Haussy, J. 1997, ACM Transactions on Mathematical Software (TOMS), 23, 209

Song, M. T., Fang, C., Tang, Y. H., Wu, S. T., \& Zhang, Y. A. 2006, ApJ, 649, 1084

Teuber, D., Tandberg-Hanssen, E., \& Hagyard, M. J. 1977, Sol. Phys., 53, 97
Titov, V. S., \& Démoulin, P. 1999, A\&A, 351, 707

Titov, V. S., Priest, E. R., \& Démoulin, P. 1993, A\&A, 276, 564

Valori, G., Kliem, B., \& Fuhrmann, M. 2007, Sol. Phys., in press

Valori, G., Kliem, B., \& Keppens, R. 2005, A\&A, 433, 335

Wheatland, M. S. 2004, Sol. Phys., 222, 247

Wheatland, M. S. 2006, Sol. Phys., 238, 29

Wheatland, M. S., Sturrock, P. A., \& Roumeliotis, G. 2000, ApJ, 540, 1150

Wiegelmann, T. 2004, Sol. Phys., 219, 87

Wiegelmann, T. 2007, Sol. Phys., 240, 227

Wiegelmann, T., \& Inhester, B. 2003, Sol. Phys., 214, 287

Wiegelmann, T., \& Neukirch, T. 2006, A\&A, 457, 1053

Wiegelmann, T., Inhester, B., \& Sakurai, T. 2006, Sol. Phys., 233, 215

Wu, S. T., Chang, H. M., \& Hagyard, M. J. 1985, in Measurements of Solar Vector Magnetic Fields, ed. M. J. Hagyard, NASA Conf. Publ., 2374, 17

Wu, S. T., Sun, M. T., Chang, H. M., Hagyard, M. J., \& Gary, G. A. 1990, ApJ, 362, 698

Yan, Y. 2003, Space Sci. Rev., 107, 119

Yan, Y., \& Li, Z. 2006, ApJ, 638, 1162

Yan, Y., \& Sakurai, T. 1997, Sol. Phys., 174, 65

Yan, Y., \& Sakurai, T. 2000, Sol. Phys., 195, 89

Yang, W. H., Sturrock, P. A., \& Antiochos, S. K. 1986, ApJ, 309, 383 